\title{
Fragmentation processes in impact of spheres
}

\author{
H. A. Carmona, ${ }^{1,2}$ F. K. Wittel, ${ }^{2}$ F. Kun, ${ }^{3}$ and H. J. Herrmann ${ }^{2,4}$ \\ ${ }^{1}$ Centro de Ciências e Tecnologia, Universidade Estadual do Ceará, 60740-903 Fortaleza, Ceará, Brazil \\ ${ }^{2}$ Computational Physics IfB, HIF, ETH, Hönggerberg, 8093 Zürich, Switzerland \\ ${ }^{3}$ Department of Theoretical Physics, University of Debrecen, P. O. Box:5, H-4010 Debrecen, Hungary \\ ${ }^{4}$ Departamento de Física, Universidade Federal do Ceará, 60451-970 Fortaleza, Ceará, Brazil
}

(Received 18 September 2007; published 9 May 2008)

\begin{abstract}
We study the brittle fragmentation of spheres by using a three-dimensional discrete element model. Large scale computer simulations are performed with a model that consists of agglomerates of many particles, interconnected by beam-truss elements. We focus on the detailed development of the fragmentation process and study several fragmentation mechanisms. The evolution of meridional cracks is studied in detail. These cracks are found to initiate in the inside of the specimen with quasiperiodic angular distribution. The fragments that are formed when these cracks penetrate the specimen surface give a broad peak in the fragment mass distribution for large fragments that can be fitted by a two-parameter Weibull distribution. This mechanism can only be observed in three-dimensional models or experiments. The results prove to be independent of the degree of disorder in the model. Our results significantly improve the understanding of the fragmentation process for impact fracture since besides reproducing the experimental observations of fragment shapes, impact energy dependence, and mass distribution, we also have full access to the failure conditions and evolution.
\end{abstract}

DOI: 10.1103/PhysRevE.77.051302 PACS number(s): 45.05.+x, 05.10. $-\mathrm{a}, 46.50 .+\mathrm{a}, 62.20 . \mathrm{M}-$

\section{INTRODUCTION}

Comminution is a very important step in many industrial applications, for which one desires to reduce the energy necessary to achieve a given size reduction and minimizing the amount of fine powder resulting from the fragmentation process. Therefore a large amount of research has already been carried out to predict the outcome of fragmentation processes. Today the mechanisms involved in the initiation and propagation of single cracks are fairly well understood, and statistical models have been applied to describe macroscopic fragmentation $[1,2]$. However, when it comes to complex fragmentation processes with dynamic growth of many competing cracks in three-dimensional (3D) space, much less is understood. Today, computers allow for 3D simulations with many thousands of particles and interaction forces that are more realistic than simple central potentials. These give a good refined insight of what is really happening inside the system, and how the predicted outcome of the fragmentation process depends on the system properties.

Experimental and numerical studies of the fragmentation of single brittle spheres have been largely applied to understand the elementary processes that govern comminution [3-22]. Experiments that were carried out in the 1960s analyzed the fragment mass and size distributions [3-5] with the striking result that the mass distribution in the range of small fragments follows a power law with exponents that are universal with respect to material, or the way energy is imparted to the system. Later it became clear that the exponents depend on the dimensionality of the object. These results were confirmed by numerical simulations that were mainly based on discrete element models (DEMs) [18,23-26]. For large fragment masses, deviation from the power law distribution could be modeled by introducing an exponential cutoff, and by using a bilinear or Weibull distribution [12,13,15,27-30]. Another important finding was that fragmentation is only obtained above a certain material dependent energy input
$[5,6,31]$. Numerical simulation could show that a phase transition at a critical energy exists, with the fragmentation regime above, and the fracture or damaged regime below the critical point $[18,19,21]$.

The fragmentation process itself became experimentally accessible with the availability of high speed cameras, giving a clear picture on the formation of the fragments [5-13]. Below the critical point, only slight damage can be observed, but the specimen mainly keeps its integrity. Above but close to the critical point, the specimen breaks into a small number of fragments of the shape of wedges, formed by meridional fracture planes, and additional cone-shaped fragments at the specimen-target contact point. Way above the critical point, additional oblique fracture planes develop, that further reduce the size of the wedge-shaped fragments.

Numerical simulations can recover some of these findings, but while two-dimensional simulations cannot reproduce the meridional fracture planes that are responsible for the large fragments [14,16-18,20,21], three-dimensional simulations have been restricted to relatively small systems, and have not focused their attention on the mechanisms that initiate and drive these meridional fracture planes $[15,19]$. Therefore their formation and propagation is still not clarified, although the resulting two to four spherical wedgedshaped fragments are observed for a variety of materials and impact conditions [5,8,11,20]. Arbiter et al. [5] argued, based on the analysis of high speed photographs, that fracture starts from the periphery of the contact disk between the specimen and the plane, due to the circumferential tension induced by a highly compressed cone driven into the specimen. However, their experiments did not allow access to the damage developed inside the specimen during impact. Using transparent acrylic resin, Majzoub and Chaudhri [8] observed damage initiation at the border of the contact disk, but in their experiments plastic flow and material imperfections may have a dominant role.

In this paper we present three-dimensional simulations of brittle solid spheres under impact with a hard plate. With our 
simulations, the time evolution of the fragmentation process and stress fields involved are directly accessible. We have focused our attention on the processes involved in the initiation and development of fracture, and how they lead to different regimes in the resulting fragment mass distributions. Our results can reproduce experimental observations on fragment shapes, impact energy dependence, and mass distributions, significantly improving our understanding of the fragmentation process in impact fracture.

\section{MODEL AND SIMULATION}

Discrete element models (DEMs) have been successfully used since they were introduced by Cundall and Strack to study rock mechanics [32]. Applications range from static to impact and explosive loading, using elementary particles of various shapes that are connected by different types of massless cohesive elements [14,17-20,24,33-38]. In general, Newton's equation governs the translational and rotational motion of the elements, that concentrate the whole mass. Forces and torques arise from element interactions, from the cohesive elements, volumetric forces, and of course from interaction with boundaries like walls.

Throughout this work we use a three-dimensional (3D) implementation of DEMs where the solid is represented by an assembly of spheres of two different sizes. They are connected via beam-truss elements that deform by elongation, shear, bending, and torsion. Besides carrying all the mass of the system, the spherical elements are not allowed to penetrate each other without a repulsive force acting on them. This mechanism is included in the model in order to take into account the possible interactions between formed fragments and fracture generated planes during the fragmentation of the material. The total force and moment acting on each element consists of the contact forces resulting from sphere-sphere interactions, $\vec{F}^{c}=\vec{F}^{o v}+\vec{F}^{\text {diss }}$, the stretching and bending forces $\vec{F}^{b}=\vec{F}^{e l o}+\vec{Q}$, and moments $\vec{M}^{b}$ transmitted by the beams attached.

The contact force has a repulsive term due to elastic interaction between overlapping spherical elements, which is given by the Hertz theory [39] as a function of the material Young's modulus $E^{p}$, the Poisson ratio $\nu^{p}$, and the deformation $\xi$. The force on element $j$ at a distance $\vec{r}_{i j}$ relative to element $i$ [see Fig. 1(a)] is given by

$$
\vec{F}_{j}^{o v}=\frac{4}{3} \frac{E^{p} \sqrt{R^{e f f}}}{\left(1-\nu^{2}\right)} \xi_{i j}^{3 / 2} \hat{r}_{i j},
$$

where the overlapping distance $\xi_{i j}=R_{i}+R_{i}-\left|\vec{r}_{i j}\right|$ describes the deformation of the spheres, $1 / R^{\text {eff }}=1 / R_{i}+1 / R_{j}$, and $\hat{r}_{i j}$ $=\vec{r}_{i j} /\left|\vec{r}_{i j}\right|$. The additional terms of the contact force include damping and friction forces and torques in the same way as described in Refs. $[1,18,24,40]$. The Hertzian contact law for spheres assumes a radius of curvature corresponding to the spherical elements used in the model, thus introducing an arbitrary length scale into the contact behavior of conforming surfaces. The use of spherical interactions also could lead to slightly increased fracture toughness for sliding fracture under compression. In our case, however, mode II failure is
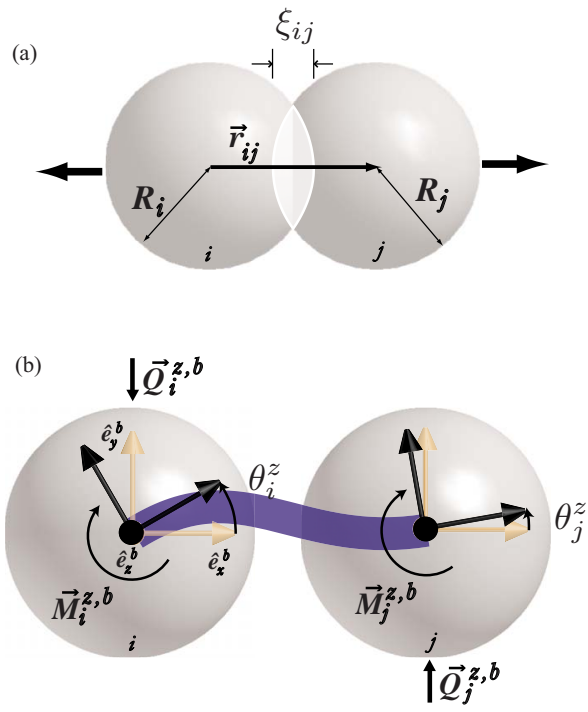

FIG. 1. (Color online) (a) Representation of the overlap interaction between two elements. (b) Typical deformation of a beam in the $x-y$ plane, showing the resulting bending and shear forces and torques. The $z$ axis is perpendicular to the image.

rare and therefore this effect should not matter here. Surfaces in contact behave nonlinearly, however, this plays no role, since the contact zones are small compared to average fragment sizes.

The 3D representation of beams used in this work is an extension of the two-dimensional case of Euler-Bernoulli beams described in Ref. [41]. In three dimensions, however, the total deformation of a beam is calculated by the superposition of elongation, torsion, as well as bending and shearing in two different planes. The restoring force acting on element $j$ connected by a beam to element $i$ due to the elongation of the beam is given by

$$
\vec{F}_{j}^{e l o}=-E^{b} A^{b} \varepsilon \hat{r}_{i j},
$$

where $E^{b}$ is the beam stiffness, $\varepsilon=\left(\left|\vec{r}_{i j}\right|-l_{0}\right) / l_{0}$, with the initial length of the beam $l_{0}$ and its cross section $A^{b}$. In this way, the restoring force is attractive for elongation and repulsive for compression of the beam-truss elements.

The flexural forces and moments transmitted by a beam are calculated from the change in the orientations on each beam end, relative to the body-fixed coordinate system of the beam $\left(\hat{e}_{x}^{b}, \hat{e}_{y}^{b}, \hat{e}_{z}^{b}\right)$. Figure $1(\mathrm{~b})$ shows a typical deformation due to rotation of both beam ends relative to the $\hat{e}_{z}^{b}$ axis, with $\hat{e}_{x}^{b}$ oriented in the direction of $\hat{r}_{i j}$. Given the angular orientations $\theta_{i}^{z}$ and $\theta_{j}^{z}$, the corresponding bending force $\vec{Q}_{j}^{z, b}$ and moment $\vec{M}_{j}^{z, b}$ for the elastic deformation of the beam are given by $[41]$ :

$$
\begin{gathered}
\vec{Q}_{j}^{z, b}=3 E^{b} I \frac{\left(\theta_{i}^{z}+\theta_{j}^{z}\right)}{L^{2}} \hat{e}_{y}^{b}, \\
\vec{M}_{j}^{z, b}=E^{b} I \frac{\left(\theta_{i}^{z}-\theta_{j}^{z}\right)}{L} \hat{e}_{z}^{b}+\left(\vec{Q}_{i}^{z, b} \times\left|\vec{r}_{i j}\right| \hat{e}_{x}^{b}\right),
\end{gathered}
$$


where $I$ is the beam moment of inertia. Corresponding equations are written for general rotations around $\hat{e}_{y}^{b}$, and the forces and moments are added up. Additional torsion moments are added to consider a relative rotation of the elements around $\hat{e}_{x}^{b}$ :

$$
\vec{M}_{j}^{x, b}=-G^{b} I^{\operatorname{tor}} \frac{\left(\theta_{j}^{x}-\theta_{i}^{x}\right)}{L} \hat{e}_{x}^{b},
$$

with $G^{b}$ and $I^{\text {tor }}$ representing the shear modulus and moment of inertia of the beams along the beam axis, respectively. The bending forces and moments are transformed to the global coordinate system before they are added to the contact, volume, and walls forces.

Beams can break in order to explicitly model damage, fracture, and failure of the solid. The imposed breaking rule takes into account breaking due to stretching and bending of a beam $[21,23,24,40,42]$, which breaks if

$$
\left(\frac{\varepsilon}{\varepsilon_{t h}}\right)^{2}+\frac{\max \left(\left|\theta_{i}\right|,\left|\theta_{j}\right|\right)}{\theta_{t h}} \geq 1,
$$

where $\varepsilon=\Delta l / l_{0}$ is the longitudinal strain, and $\theta_{i}$ and $\theta_{j}$ are the general rotation angles at the beam ends between elements $i$ and $j$, respectively. Here $\cos \theta_{i}=\hat{e}_{x}^{i b} \cdot \hat{e}_{x}^{b}$, where $\left(\hat{e}_{x}^{i b}, \hat{e}_{y}^{i b}, \hat{e}_{z}^{i b}\right)$ define the $i$-particle's orientation in the beam body-fixed coordinate system, similar calculation is performed to evaluate $\theta_{j}$. Equation (5) has the form of the von Mises yield criterion for metal plasticity [40,43]. The first part of Eq. (5) refers to the breaking of the beam through stretching and the second through bending, with $\varepsilon_{t h}$ and $\theta_{t h}$ being the respective threshold values. The introduced threshold values are taken randomly for each beam, according to the Weibull distributions:

$$
\begin{aligned}
& P\left(\varepsilon_{t h}\right)=\frac{k}{\varepsilon_{o}}\left(\frac{\varepsilon_{t h}}{\varepsilon_{o}}\right)^{k-1} \exp \left[-\left(\frac{\varepsilon_{t h}}{\varepsilon_{o}}\right)^{k}\right], \\
& P\left(\theta_{t h}\right)=\frac{k}{\theta_{o}}\left(\frac{\theta_{t h}}{\theta_{o}}\right)^{k-1} \exp \left[-\left(\frac{\theta_{t h}}{\theta_{o}}\right)^{k}\right] .
\end{aligned}
$$

Here $k, \varepsilon_{o}$, and $\theta_{o}$ are parameters of the model, controlling the width of the distributions and the average values for $\varepsilon_{t h}$ and $\theta_{t h}$, respectively. Low disorder is obtained by using large $k$ values, large disorder by small $k$. Disorder is also introduced in the model by the different beam lengths in the discretization as described below.

The time evolution of the system is followed by numerically solving the equations of motion for the translation and rotation of all elements using a sixth-order Gear predictorcorrector algorithm, and the dynamics of the rotations of the elements is described using quaternions [41,44]. The breaking rules are evaluated at each time step. The beam breaking is irreversible, which means that broken beams are excluded from the force calculations for all consecutive time steps.

\section{System formation and characterization}

Special attention needs to be given to the discretization in order to prevent artifacts arising from the system topology, like anisotropic properties, leading to nonuniform propagation of elastic waves or preferred crack paths. In our procedure we first start with 27000 spherical elements that we initially place on a cubic lattice with random velocities. The element diameters are of two different sizes, with $D_{2}$ $=0.95 D_{1}$, that are randomly assigned, leading to more or less equal fractions. Once the elements are placed, the system is left to evolve for 50000 time steps, using periodic boundary conditions, in a volume that is about eight times larger than the total volume of the elements. This way we obtain truly random and uniformly distributed positions.

To compact the elements, a centripetal constant acceleration field, directed toward the center of the simulation box, is imposed. Due to this field the elements form a nearly spherical agglomerate at the center of the box. The system is allowed to evolve until all particle velocities are reduced to nearly zero due to dissipative forces.

With the elements compacted, the next stage is to connect them by beam-truss elements. This is achieved in our model through a Delaunay triangulation of their positions. As a consequence, not only spherical elements that are initially in contact or nearly in contact with each other are connected, but the resulting beam lattice is equivalent to a discretization of the material using a dual Voronoi tessellation of the material domain $[43,45,46]$. After the bonds have been positioned, their Young's moduli are slowly increased while the centripetal field is reduced to zero. During this process the material expands to an equilibrium state, reducing the contact forces. The bond lengths and orientations are then reset so that no initial residual stresses are present in the beam lattice. The final solid fraction obtained is approximately 0.65 . We have compared impact simulations of specimens compacted as described above with specimens using random packing of spheres as reported in Ref. [47], which have no preferential direction in the packing process such as the one that could be imposed by the centripetal field. No significant difference was found in the simulation results, indicating that possible radially aligned locked-in force chains are not relevant.

Once the system is formed, the specimen is shaped to the desired geometry by removing particles and beams that are situated outside the chosen volume. The microscopic properties, namely the elastic properties of the elements and bonds, as well as the bond breaking thresholds, are chosen to attain the desired macroscopic Young's modulus, Poisson's ratio, as well as the tensile and compressive strength. Table I summarizes the input values used in the simulations presented in this paper. These were chosen to obtain macroscopic properties close to the mechanical properties of polymers like Polymethyl methacrylate (PMMA), Polyamide (PA), and nylon at low temperatures. Figure 2 displays the stress-strain curve measured by quasistatic, uniaxial tensile loading of a bar, as depicted in the inset. The microscopic and resulting macroscopic properties are resumed in Table I, for a sample size $(16 \times 8 \times 8 \mathrm{~mm})$. The experiment is performed by measuring the force required to slowly move the upper and lower surfaces (see inset of Fig. 2) at a constant strain rate of $0.004 \mathrm{~s}^{-1}$. The stress-strain curve is basically linear until the strength is reached where rapid brittle fracture of the material takes place. Oscillations in the broken specimen fractions 
TABLE I. Micro- and macroscopic material model properties.

\begin{tabular}{|c|c|c|c|}
\hline \multicolumn{4}{|c|}{$\begin{array}{l}\text { Typical model properties (DEM): } \\
\text { Beams: }\end{array}$} \\
\hline Stiffness & $E^{b} / G^{b}$ & 6 & $\mathrm{GPa}$ \\
\hline Average length & $L$ & 0.5 & $\mathrm{~mm}$ \\
\hline Diameter & $d$ & 0.5 & $\mathrm{~mm}$ \\
\hline Strain threshold & $\varepsilon_{0}$ & 0.02 & - \\
\hline Bending threshold & $\theta_{0}$ & 3 & $\circ$ \\
\hline Shape parameter & $k$ & $3 / 10$ & - \\
\hline \multicolumn{4}{|c|}{ Particles: } \\
\hline Stiffness & $E^{p}$ & 3 & $\mathrm{GPa}$ \\
\hline Diameter & $D_{1}$ & 0.5 & $\mathrm{~mm}$ \\
\hline Density & $\rho$ & 3000 & $\mathrm{~kg} / \mathrm{m}^{3}$ \\
\hline \multicolumn{4}{|c|}{ Hard plate: } \\
\hline Stiffness & $E^{w}$ & 70 & $\mathrm{GPa}$ \\
\hline \multicolumn{4}{|c|}{ Interaction: } \\
\hline Friction coefficient & $\mu$ & 1 & - \\
\hline Damping coefficient & $\gamma_{n}$ & 0.25 & $\mathrm{~s}^{-1}$ \\
\hline Friction coefficient & $\gamma_{t}$ & 0.05 & $\mathrm{~s}^{-1}$ \\
\hline \multicolumn{4}{|c|}{ System: } \\
\hline Time increment & $\Delta t$ & $1 \mathrm{e}-8$ & $\mathrm{~s}$ \\
\hline Number of particles & $N^{p}$ & 22013 & - \\
\hline Number of beams & $N^{b}$ & 135948 & - \\
\hline Solid fraction & & 0.65 & - \\
\hline Sphere diameter & $D$ & 16 & $\mathrm{~mm}$ \\
\hline \multicolumn{4}{|c|}{ Macroscopic properties (DEM): } \\
\hline System stiffness & $E$ & $7.4 \pm 0.5$ & $\mathrm{GPa}$ \\
\hline Poisson's ratio & $\nu$ & 0.2 & - \\
\hline Density & $\rho$ & 1920 & $\mathrm{~kg} / \mathrm{m}^{3}$ \\
\hline System strength & $\begin{array}{c}\sigma_{c} \\
\text { Comparison: }\end{array}$ & 110 & $\mathrm{MPa}$ \\
\hline & DEM & FEM & \\
\hline $\begin{array}{l}\text { Longitudinal } \\
\text { Wave speed }\end{array}$ & $2210 \pm 100$ & $2270 \pm 20$ & $\mathrm{~m} / \mathrm{s}$ \\
\hline Contact time & 31.4 & 31.4 & $\mu \mathrm{s}$ \\
\hline
\end{tabular}

can be seen after the system is completely unloaded due to elastic waves. The Young's modulus measured from the slope of the curve is $7.4 \pm 0.5 \mathrm{GPa}$, is presented along with other macroscopic properties of the material in Table I. Although not shown in Fig. 2, an identical Young's modulus is obtained under uniaxial compression.

In order to simulate the impact of a sphere on a frictionless hard plate, a spherical specimen with diameter $D$ $=16 \mathrm{~mm}$ is constructed, and a fixed plane with Young's modulus $70 \mathrm{GPa}$ is added to the simulation. The spherical specimen has a total of approximately 22000 elements, with around 32 across the sample diameter. The contact interaction between the elements and the plate is identical to the element-element contact interaction, only with $\xi=R_{i}-r_{i p}$, where $r_{i p}$ is the distance between the particle center and the plate.

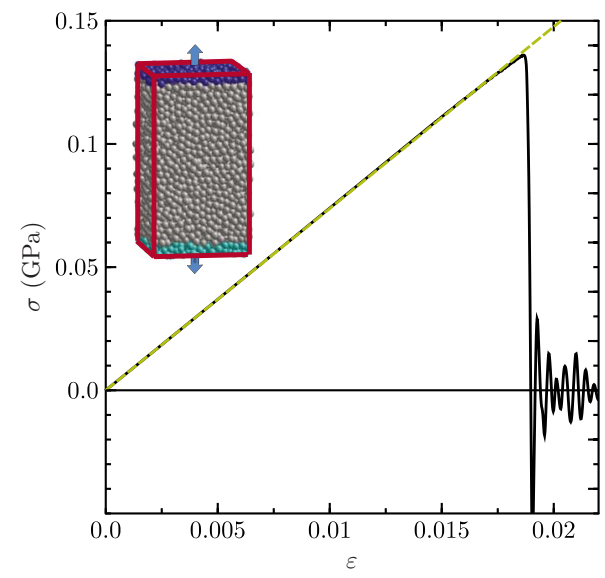

FIG. 2. (Color online) Stress-strain curve for specimen under quasistatic loading. The inset shows the load geometry. Abrupt brittle fracture behavior can be observed at about $\varepsilon=0.019$.

Friction between the specimen and the plate is excluded in the present study. Preliminary tests for a few friction coefficients, however, did not show any influence in our results. This is probably due to the fact that for the geometry used in our simulations, highly hydrostatic compressive stresses are induced near the contact point and very little damage occurs in this region during the contact period; see for example Fig. 4(a). The specimen is placed close to the plate with an impact velocity $v_{i}$, in the negative $z$ direction, assigned to all its composing elements. The computation continues until no additional bonds are broken for at least $50 \mu$ s.

For comparative reasons we calculate the evolution of the stress field using an explicit finite element (FE) analysis. The FE model is composed of axisymmetric, linear four-node elements with macroscopic properties taken from the results of the DEM simulations (see Table I). Along the central axis through the sphere and ground plate, symmetry boundary conditions are imposed, the bottom of the target plate is encrusted, and contact surfaces for the sphere and plate are defined. Figure 3(a) shows a comparison between the impact simulation using our DEM model and a finite element model simulation. In Fig. 3(a), the DEM elements are colored according to the amplitude of their accelerations to show the propagation of a longitudinal shock wave that was initiated at the contact point. The wave speed can be estimated to be approximately $2200 \pm 100 \mathrm{~m} / \mathrm{s}$, which is consistent with the Young's modulus of the material derived from Fig. 2 and its density. The time evolution of the potential energy stored in the system is compared in Fig. 3(b), showing excellent quantitative agreement between the two models. After the characterization of the system properties we allow for the cohesive elements to fail in order to study the fragmentation properties.

\section{FRAGMENTATION MECHANISMS}

In this section we explore the different fragmentation mechanisms in the order of occurrence and increasing energy input. The first yield that arises in the material is diffuse damage that occurs in the region above the contact disk. It 
(a)

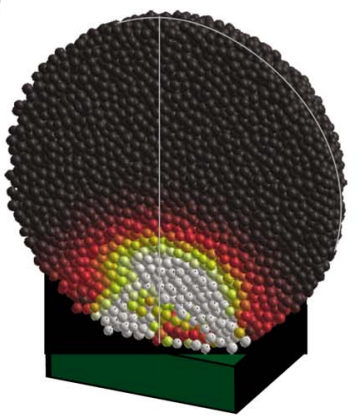

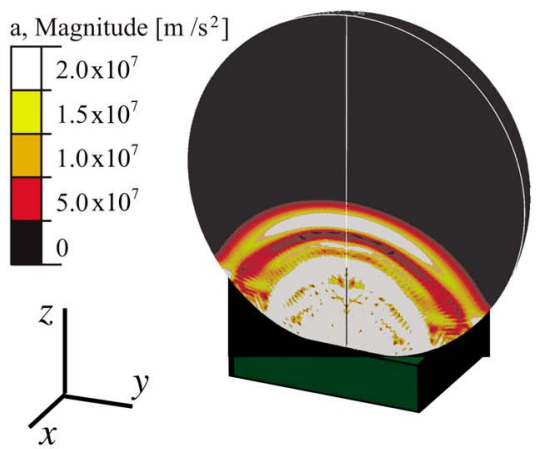

(b)

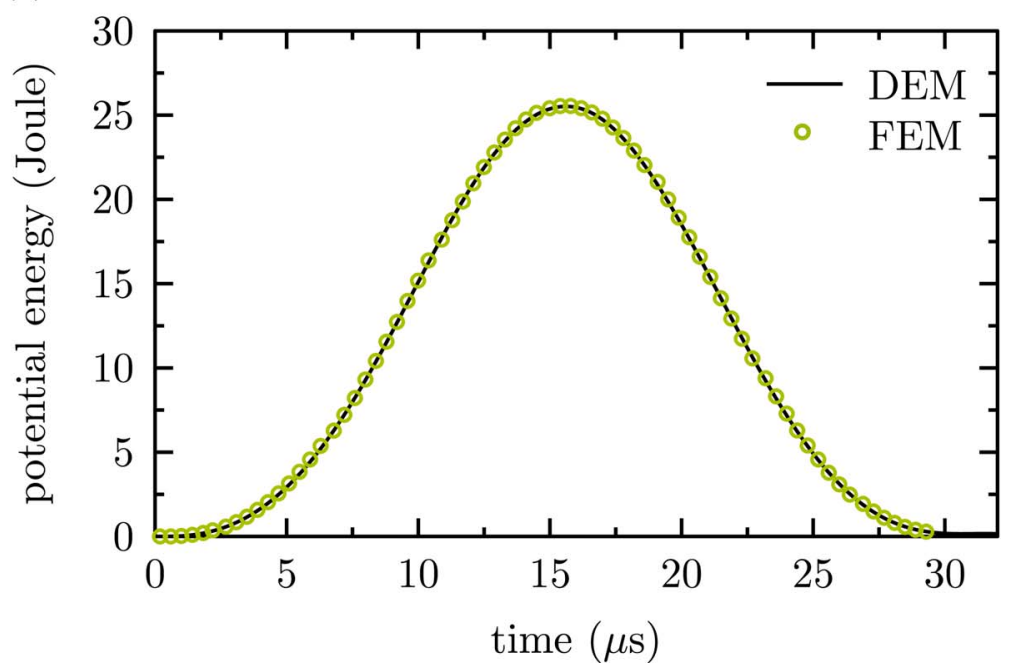

FIG. 3. (Color online) (a) Comparison of deformations and shock-wave propagation obtained between DEM and FEM simulations for $v_{i}$ $=117 \mathrm{~m} / \mathrm{s}$. (b) Time evolution of the elastic potential energy stored in the system for the same velocity obtained by DEM (solid line) and FEM (dashed line) simulations. can be seen from Fig. 4(a) that this damage region is centered in the load axis, at a distance approximately $D / 4$ from the plane.

We can see a strong correlation of the position of the diffuse cracking in the DEM results [Fig. 4(a)] with the location of a region with a biaxial stress state in the $x-y$ plane and a superimposed compression in the $z$ direction, as calculated using FEM [Fig. 4(b)], also in agreement with experimental results reported in Ref. [6]. This result, along with the one presented in Fig. 3, suggests that the use of threedimensional beams, as compared with the use of simple springs, despite the reduced number of degrees of freedom in the breaking criterion, could recover quite well the influence of complex stress states in the crack formation in a more precise way.

As time evolves, meridional cracks start to appear. The origination of this type of cracks is explored in Fig. 5, where we plot in Fig. 5(a) the positions of the broken bonds in two different projections, showing well defined meridional crack planes that propagate toward the lateral and upper free surfaces of the specimen. In Fig. 5(b) we plot the angular distribution of the broken bonds for different times. Here $g(\theta)$ is the probability of finding two broken bonds with an angular separation $\theta$. Note that their positions are projected into the plane perpendicular to the load axis. The evident peaks in $g(\theta)$ are a clear indication that the cracks are meridional planes that include the load axis. In this particular case, the cracks are separated by an average angle of about $60^{\circ}$, and they become evident $13-15 \mu \mathrm{s}$ after impact $\left(v_{i}\right.$ $=120 \mathrm{~m} / \mathrm{s}$ ).
In order to understand what governs the orientation and angular separation of these meridional cracks we performed many different realizations with different seeds of the random number generator and impact points. For all cases the orientation of the cracks can change, but not their average angular separation. We observe that for strong disorder [Eq. (6a) and (6b)], a larger amount of uncorrelated damage occurs, but the average angular separation of the primary cracks does not change. This suggests that the formation of these cracks arises due to a combination of the existence of local disorder and the stress field in the material, but does not depend on the degree of disorder.

As we can see from the FE calculations and from the damage orientation correlation plot (Fig. 5) inside of the uni- (a)

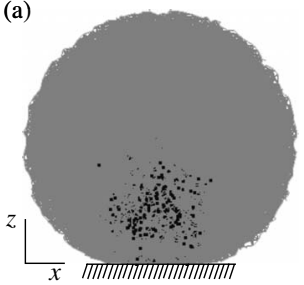

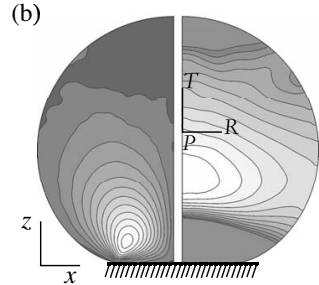

FIG. 4. Initial damage due to biaxial stress state. (a) Vertical cut through the center of the sphere from the DEM simulation showing broken bonds represented by dark color. (b) Stress fields calculated with FEM model. Left side are shear stresses in global coordinates from 0 to $-400 \mathrm{MPa}$ (black to white) while the right side shows circumferential stresses in local spherical coordinates with the center in the sphere center ranging from 0 to $130 \mathrm{MPa}$ (black to white). 

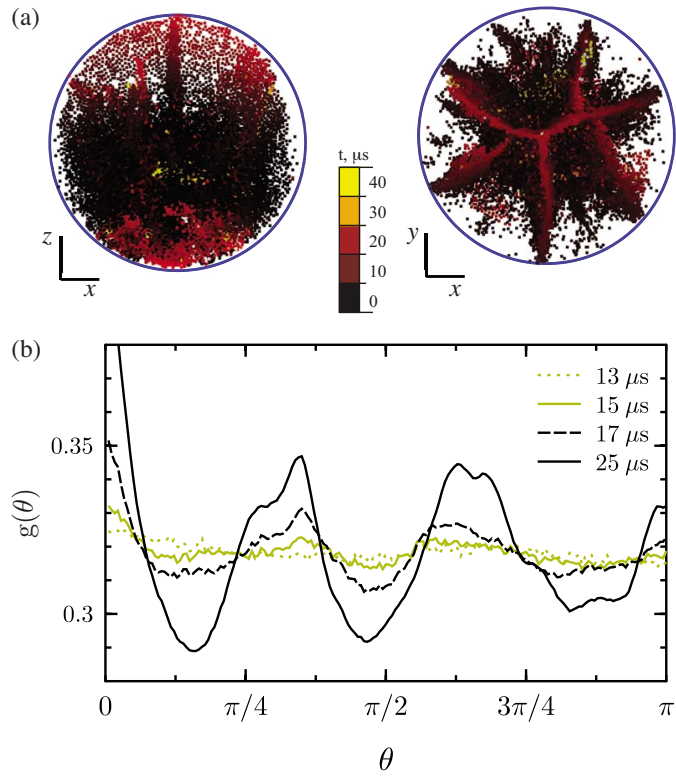

FIG. 5. (Color online) (a) Colored dots display the positions of the broken bonds according to the time of breaking. (b) Angular distribution function of broken bonds as a function of the angular separation when their positions are projected into the plane perpendicular to the load axis.

form biaxial tensile zone, no preferred crack orientation is evidenced. Many microcracks weaken this material zone, decreasing the effective stiffness of the core. Around the weakened core the material is intact and under high circumferential stresses. It is in this ring-shaped zone that we observe to be the onset of the meridional cracks when we trace them back. For increasing impact velocity we observe a decrease in the angular separation of crack planes and thus more wedge-shaped fragments. Therefore this fragment formation mechanism cannot be explained by a quasistatic stress analysis. The observation is in agreement with experimental findings and can be explained by the basic ideas of Mott's fragmentation theory for expanding rings [48]. Due to the stress release front for circumferential stresses: once a meridional crack forms, stress is released in its neighborhood; the fractured regions spread with a constant velocity and the probability for fracture in neighboring regions decreases. On the other hand, in the unstressed regions, the strain still increases, and so does the fracture probability along with it. The average size of the wedge-shaped fragments therefore is determined by the relationship between the velocity of the stress release wave and the rate at which cracks nucleate. Thus the higher the strain rate, the higher the crack nucleation rate and the more fragments are formed. We measured the strain rate at different positions inside the biaxially loaded zone, finding a clear correlation between impact velocity and strain rate. Even though we fragment a compact sphere and not a ring, when it comes to the formation of meridional cracks, we observe that they form in a ringshaped region and that Mott's theory can qualitatively explain the decrease of angular separation between wedgeshaped fragments with increasing impact velocity. If enough energy is still available, some of the meridional plane cracks grow outwards and upwards, breaking the sample into
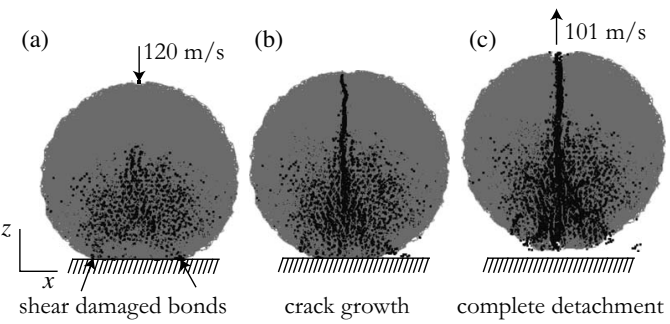

FIG. 6. Vertical meridional cut of the sphere at different stages during impact, showing the separation of lower fragments. (a) Formation of a ring of broken bonds due to shear failure. (b) These broken bonds evolve into cracks that propagate inside the sample. (c) Finally these cracks lead to the detachment of the lower fragments.

wedge-shaped fragments that resemble orange slices.

As the sphere continues moving toward the plate, a ring of broken bonds forms at the border of the contact disk due to shear failure [compare Fig. 6(a)]. When the sphere begins to detach from the plate, the cone has been formed by high shear stresses in the contact zone [see Fig. 4(b) left] by a ring crack that was able to grow from the surface to the inside of the material under approximately $45^{\circ}$ [Fig. 6(b)]. It detaches, leaving a small number of cone-shaped fragments that have a smaller rebound velocity than the rest of the fragments due to dissipated elastic energy [Fig. 6(c)].

Oblique plane cracks may still break the large fragments further, if the initial energy given to the system is high enough. Therefore they are called secondary cracks. Figure 7(a) shows a vertical meridional cut of a sample where these cracks can be seen. The intact bonds are colored according to the final fragment to which they belong.

These secondary cracks are very similar to the oblique cracks observed in 2D simulations [14,16,21]. For comparison we show in Fig. 7(b) the crack patterns obtained from a 2D DEM simulation that uses polygons as elementary particles.

The oblique cracks in two dimensions are, however, primary cracks, originated from the tensile stresses induced by impact [16], while secondary cracks are azimuthal cracks, roughly perpendicular to them, concentrated near the contact point. From this point of view the direct analog of the 2D oblique cracks are, in fact, the meridional cracks in three dimensions.

Our simulations clearly show that the 3D oblique cracks appear as a secondary mechanism that can only occur after
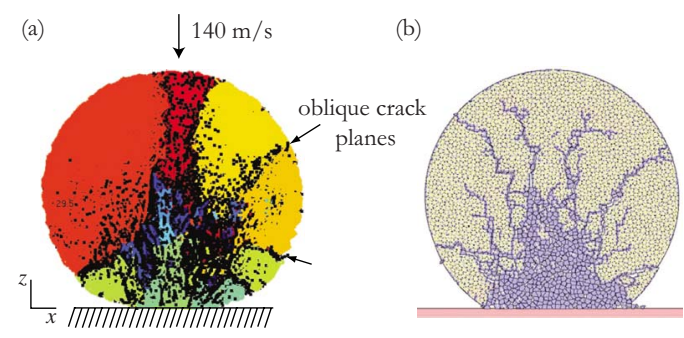

FIG. 7. (Color online) (a) DEM simulation at $v_{i}=140 \mathrm{~m} / \mathrm{s}$ exemplifying the secondary cracks. The bonds are colored according to the final fragment they belong to. (b) $2 \mathrm{D}$ simulations using polygons as elementary particles [21]. 

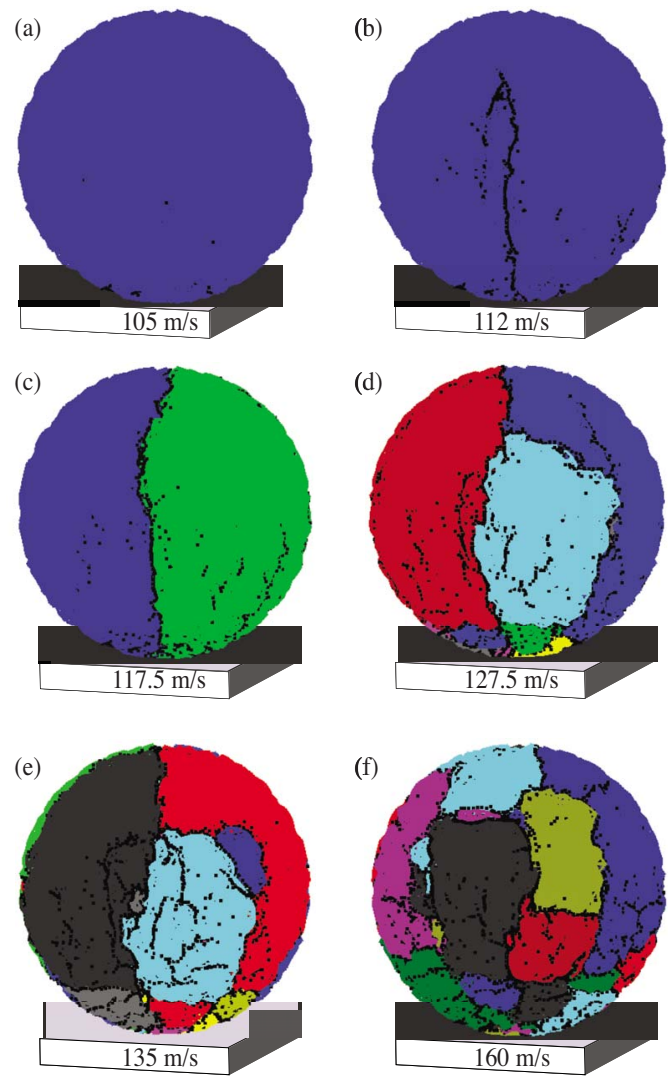

FIG. 8. (Color online) Front view of the reconstructed spheres, showing the final crack patterns at the surfaces for different initial velocities. Gray dots are placed at the positions of broken beams while different colors are chosen for different fragments.

the meridional cracks have been formed. This behavior can be seen as an analog to the mechanism II impact breakage in Ref. [16], which is related to the formation of secondary cracks as a consequence of the buckling of the primary elongated fragments in two dimensions. Our results show that here also the secondary cracks result from the bucking of the long wedge-shaped fragments originated from the primary cracks. The concentration of these cracks near the contact point reinforces this point of view.

\section{FRAGMENTATION REGIMES}

The amount of energy necessary to fragment a material is a parameter that is very important for practical applications in comminution. In fragmentation experiments two distinct regimes for damage and fragmentation can be identified depending on the impact energy: below a critical energy $[4,6,19]$ damage takes place, while above fragments are formed. Figure 8 compares the final crack patterns after impact with different initial velocities. The intact bounds are colored according to the final fragment to which they belong, and gray dots display the positions of broken bonds. The fragments have been reassembled to their initial positions to provide a clearer picture of the resulting crack patterns. For the smaller impact velocities it is possible to identify meridional cracks that reach the sample surface above the contact

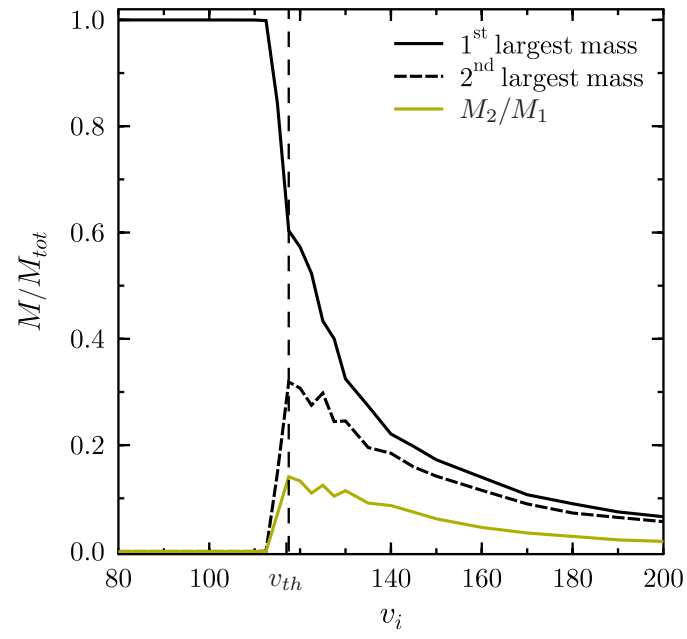

FIG. 9. (Color online) The mass of first and second largest fragment and the average fragment mass as a function of the impact velocity.

point, but fragmentation is not complete and one large piece remains [Figs. 8(a) and 8(b)]. We call these meridional cracks primary cracks, since as one increases the initial energy given to the system, some of them are the first to reach the top free surface of the sphere, fragmenting the material into a few large pieces, typically two or three fragments with wedge shapes [Fig. 8(c)]. When we increase the initial energy secondary oblique plane cracks break the orange-sliceshaped fragments further [Fig. 8(d)]. Additional increase in the impact velocity causes more secondary cracks and consequently the reduction of the fragment sizes [Figs. 8(e) and $8(\mathrm{f})]$.

The shape and number of large fragments resulting from the numerical model for smaller impact energies as well as the location and orientation of oblique secondary cracks for larger energies are in agreement with experimental findings $[11,12,20]$.

We can identify that for velocities smaller then a threshold value, the sample is damaged by the impact but not fragmented. This threshold velocity for fragmentation has been found experimentally and numerically $[6,18,21]$. In particular, it has been found from 2D simulations that a continuous phase transition from damaged to fragmented outcome of impact fragmentation can be tuned by varying the initial energy imparted to the system $[18,21]$.

Following the analysis in Refs. $[18,21]$ the final state of the system after impact is analyzed by observing the evolution of the mass of the two largest fragments, as well as the average fragment size (shown in Fig. 9). The average mass $M_{2} / M_{1}$, with $M_{k}=\Sigma_{i}^{N_{f}} M_{i}^{k}-M_{\max }^{k}$ excludes the largest fragment. It can be observed that below the threshold value $v_{\text {th }}$ $=115 \mathrm{~m} / \mathrm{s}$ the largest fragment has almost the total mass of the system, while the second largest is orders of magnitude smaller. This behavior implies that for $v_{i}<v_{t h}$ the system does not fragment, it only gets damaged. For velocities larger than $v_{t h}$ the mass of the largest fragment decreases rapidly. The second largest and average fragment masses increase, having their maximum at $117.5 \mathrm{~m} / \mathrm{s}$ for this material strength.

The results shown in Fig. 9 are in very good qualitative agreement with those obtained from simulations in different 

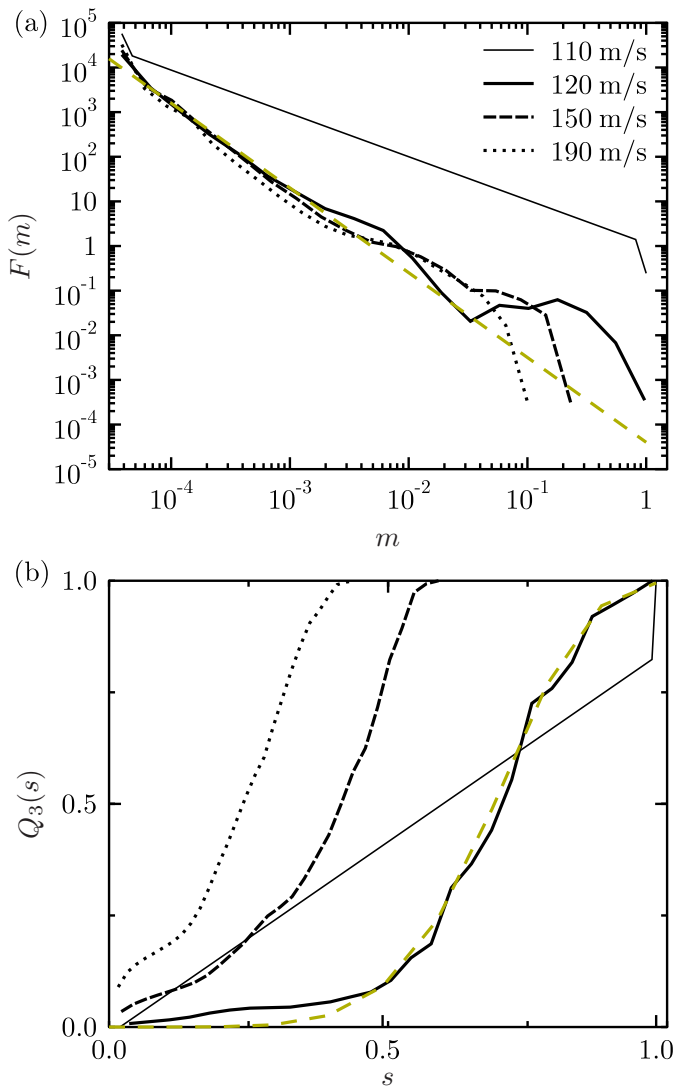

FIG. 10. (Color online) (a) Fragment mass distribution for different initial velocities. The straight line corresponds to a power law with exponent -1.9 (b) Fragment size distribution weighted by mass for initial velocities with identical legend as above.

geometries and load conditions $[18,21,49]$, indicating that our model shows a phase transition from a damaged to a fragmented state.

\section{RESULTING FRAGMENT MASS DISTRIBUTION}

One of the first and still most important characterizations for fragmentation processes are fragment mass distributions. Experimental and numerical studies on fragmentation show that the mass distribution follows a power law in the range of small fragments, whose exponent depends on the fragmentation mechanisms, while the mass distribution for large fragments is usually represented by an exponential cutoff of the power law. The fragment mass distributions that are obtained from our three-dimensional simulations are given in Fig. 10(a) for different impact velocities $v_{i}$. Here $F(m)$ represents the probability density of finding a fragment with mass $m$ between $m$ and $m+\Delta m$, where $m$ is the fragment mass normalized by the total mass of the sphere $M_{t o t}$. The values are averaged over 36 simulations, changing the random breaking thresholds and randomly rotating the sample to obtain different impact points. For velocities below the critical velocity $v_{t h}$ of our model, the fragment mass distribution shows a peak at low fragment masses, corresponding to some small fragments. The pronounced isolated peaks near the total mass of the system correspond to large damaged, but still

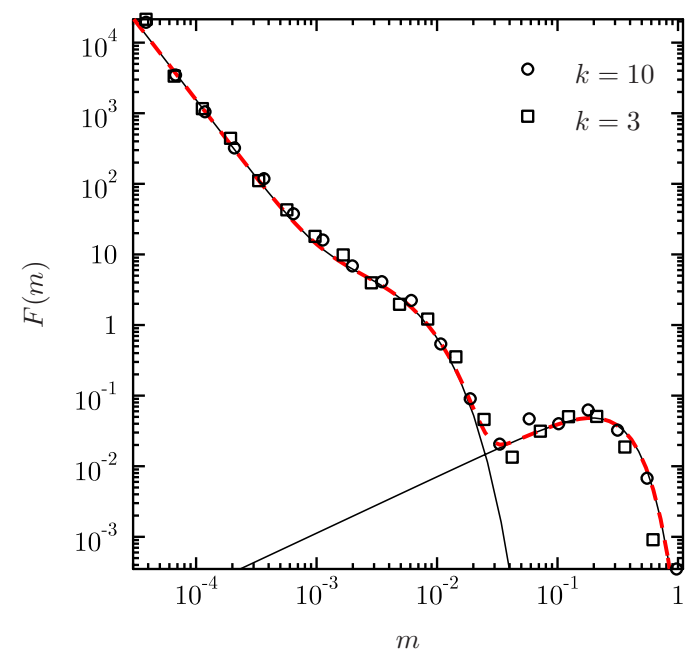

FIG. 11. (Color online) Fragment mass distribution at $v$ $=122.5 \mathrm{~m} / \mathrm{s}$ for different disorder in the bond breaking thresholds. The solid lines correspond to a power law with an exponential cutoff for lower masses and the Weibull distribution for large masses [Eq. (8)].

unbroken system [see also Figs. 8(a) and 8(b)]. Fragments at intermediate mass range are not found for small initial velocities. At and above $v_{t h}$, the fragment mass distribution exhibits a power law dependence for intermediate masses, $F(m) \sim m^{-\tau}$ [dashed line in Fig. 10(a)], with $\tau=1.9 \pm 0.2$ [50,51], and a broad maximum can be observed in the histogram for large fragments, indicating that these fragments have their origin in mechanisms distinct from the ones that form small fragments. Figure 10(b) shows the cumulative size distribution of the fragments weighted by mass, $Q_{3}$, for the same velocities. $Q_{3}$ is calculated by summing the mass of all the fragments smaller then a given size $s$. The size of a fragment is estimated as the diameter of a sphere with identical mass, the values are normalized by the sample diameter. By this representation the large fragments are better resolved. We can see that the shape of the size distribution for large fragments can be described by a two-parameter Weibull distribution, $Q_{3}(s)=1-\exp \left[-\left(s / s_{c}\right)^{k_{s}}\right]$ [dashed line in Fig. 10 (b), with $s_{c}=0.75$ and $\left.k_{s}=5.8\right]$. The Weibull distribution is used here since it has been empirically found to describe a large number of fracture experiments, especially for brittle materials [29]. With increasing initial velocity, the average fragment size shifts toward smaller values, also in agreement with experimental findings from Refs. [13,30].

The local maximum in the fragment mass distribution for large fragments represents those fragments that are formed by the meridional cracks. As we can observe from Fig. 11, the fragment mass distribution is independent of the amount of disorder or material that the specimen is composed of [ $k$ is in the breaking thresholds distributions in Eq. (8)]. Near the critical velocity $v_{t h}$ we can identify two main parts in the fragment mass distribution. Near the critical velocity two distinct regimes of the fragment mass distribution can be identified. For $m$ up to around 1/40 (approximately 550 elements), the fragment mass distribution can be well described by the form 


$$
F(m) \sim(1-\beta) m^{-\tau} \exp \left(-m / \bar{m}_{0}\right)+\beta \exp \left(-m / \bar{m}_{1}\right)
$$

which has recently been proposed by Astrom et al. [2,52]. This functional form has successfully been applied to describe experimental results and that of computer simulations $[2,49,52,53]$. The first term of Eq. (7) is associated to the branching-merging process of instable cracks, while the second one originates from the Poissonian nucleation process of the first dominating cracks. The parameter $\beta$ controls the relative importance of the two mechanisms, furthermore, the exponent $\tau$ only depends on the dimensionality of the system. The broad maximum for even larger $m$ can also be described by a two-parameter Weibull distribution as discussed above,

$$
F(m) \sim\left(\frac{k_{l}}{\bar{m}_{l}}\right)\left(\frac{m}{\bar{m}_{l}}\right)^{k_{l}-1} \exp \left[-\left(\frac{m}{\bar{m}_{l}}\right)^{k_{l}}\right] .
$$

In Fig. 11 the Eqs. (7) and (8) are plotted separately and the dashed line corresponds to a fit to the data using $\bar{m}_{0}$ $=0.001 \pm 0.001, \bar{m}_{1}=0.004 \pm 0.001, \bar{m}_{l}=0.3 \pm 0.02$, and $k_{l}$ $=1.9 \pm 0.1$. The good quality of the fit allows for the estimation of the exponent of the power-law distribution in the small fragment mass range $\tau=2.2 \pm 0.2$.

For the material parameters used in our calculations the primary cracks have an angular distribution with an average separation from $45^{\circ}$ to $60^{\circ}$. Therefore the mass of a fragment resulting from these plane meridional cracks is of the order of $10 \%$ of the sample mass, although typically only two to four cracks actually reach the surface breaking the material. This estimate corresponds to the range of masses that present the broad peak in the fragment mass distribution. This feature of the mass distribution function is not observed in the results of $2 \mathrm{D}$ simulations $[18,21]$ or $3 \mathrm{D}$ simulations of shell fragmentation [49,54], where obviously meridional cracks cannot exist.

\section{CONCLUSIONS}

We studied a brittle, disordered fragmenting solid sphere. We performed 3D DEM simulations with 3D beam-truss elements for the particle cohesion. Due to this computationally more laborious approach as compared to previous works, we were able to obtain a clearer picture of the fragmentation process, the evolution of fragmentation mechanisms, and its consequences for the fragment mass distribution. To get a clearer insight into the fracture initiation, we used continuum solutions for the stress field, obtained by the finite element method. We were able to show that 2D simulations for fragmenting systems are not capable of capturing fragmentation by meridional cracks, that are the primary cracking mechanism. We found that cracks form inside the sample in the region above a compressive cone long time before they are experimentally observable from the outside, if at all. They grow to form meridional fracture planes that result in a small number of large wedge-shaped fragments, typically two to four. The increasing tensile radial and circumferential stress in the ring-shaped region above the contact plane gives rise to meridional cracks. The decrease in the angular separation between these cracks could be explained by the Mott fragmentation model. Some of these cracks grow to form the meridional fracture planes that break the material in a small number of large fragments, and it is only then that they become visible in experiments.

The resulting mass distribution of the fragments presents a power law regime for small fragments and a broad peak for large fragments that can be fitted with a two-parameter Weibull distribution, in agreement with experimental results $[10,13,29,30]$. The fragment mass distribution is quite robust, independent of the macroscopic material properties such as material strength and disorder distribution. Only the large fragment range of the mass distribution happens to be energy dependent, due to additional fragmentation processes that arise as one increases the impact energy.

Even though our results are valid for various materials with disorder, they are limited to the class of brittle, heterogeneous media. Extensions to ductile materials are in progress. Another class of interesting questions deal with the problem of size effects, the influence of polydisperse particles or the stiffness contrast of particles, and beam elements. The ability of the model to reproduce well defined crack planes also opens up the possibility to study other crack propagation problems in three dimensions. For technological applications questions about the influence of target shapes and the optimization potential to obtain desired fragment size distributions or to reduce impact energies are of broad interest.

\section{ACKNOWLEDGMENTS}

We thank the German Federation of Industrial Research Associations "Otto von Guericke" e.V. (AiF) for financial support, under Grant No. 14516N from the German Federal Ministry of Economics and Technology (BMWI). F.K. was supported by OTKA T049209. We thank Dr. Jan Blömer and Professor José Andrade Soares Jr. for helpful discussions.
[1] Statistical Models for the Fracture of Disordered Media, edited by H. J. Herrmann and S. Roux (North-Holland, Amsterdam, 1990).

[2] J. A. Åström, Adv. Phys. 55, 247 (2006).

[3] J. J. Gilvarry and B. H. Bergstrom, J. Appl. Phys. 32, 400 (1961).

[4] J. J. Gilvarry and B. H. Bergstrom, J. Appl. Phys. 33, 3211
(1962).

[5] N. Arbiter, C. C. Harris, and G. A. Stamboltzis, Trans. Soc. Min. Eng. AIME 244, 118 (1969).

[6] E. W. Andrews and K. S. Kim, Mech. Mater. 29, 161 (1998).

[7] J. Tomas, M. Schreier, T. Groger, and S. Ehlers, Powder Technol. 105, 39 (1999).

[8] R. Majzoub and M. M. Chaudhri, Philos. Mag. Lett. 80, 387 
(2000).

[9] K. T. Chau, X. X. Wei, R. H. C. Wong, and T. X. Yu, Mech. Mater. 32, 543 (2000).

[10] A. D. Salman, C. A. Biggs, J. Fu, I. Angyal, M. Szabo, and M. J. Hounslow, Powder Technol. 128, 36 (2002).

[11] S. Z. Wu, K. T. Chau, and T. X. Yu, Powder Technol. 143, 41 (2004).

[12] W. Schubert, M. Khanal, and J. Tomas, Int. J. Miner. Process. 75, 41 (2005).

[13] S. Antonyuk, M. Khanal, J. Tomas, S. Heinrich, and L. Morl, Chem. Eng. Process. 45, 838 (2006).

[14] A. V. Potapov, M. A. Hopkins, and C. S. Campbell, Int. J. Mod. Phys. C 6, 371 (1995).

[15] A. V. Potapov and C. S. Campbell, Int. J. Mod. Phys. C 7, 717 (1996)

[16] A. V. Potapov and C. S. Campbell, Powder Technol. 93, 13 (1997).

[17] C. Thornton, K. K. Yin, and M. J. Adams, J. Phys. D 29, 424 (1996).

[18] F. Kun and H. J. Herrmann, Phys. Rev. E 59, 2623 (1999).

[19] C. Thornton, M. T. Ciomocos, and M. J. Adams, Powder Technol. 105, 74 (1999).

[20] M. Khanal, W. Schubert, and J. Tomas, Granular Matter 5, 177 (2004).

[21] B. Behera, F. Kun, S. McNamara, and H. J. Herrmann, J. Phys.: Condens. Matter 17, S2439 (2005).

[22] H. J. Herrmann, F. K. Wittel, and F. Kun, Physica A 371, 59 (2006).

[23] F. Kun and H. J. Herrmann, Int. J. Mod. Phys. C 7, 837 (1996).

[24] F. Kun and H. J. Herrmann, Comput. Methods Appl. Mech. Eng. 138, 3 (1996).

[25] A. Diehl, H. A. Carmona, L. E. Araripe, J. S. Andrade, and G. A. Farias, Phys. Rev. E 62, 4742 (2000).

[26] J. A. Åström, B. L. Holian, and J. Timonen, Phys. Rev. Lett. 84, 3061 (2000).

[27] L. Oddershede, P. Dimon, and J. Bohr, Phys. Rev. Lett. 71, 3107 (1993).

[28] A. Meibom and I. Balslev, Phys. Rev. Lett. 76, 2492 (1996).

[29] C. S. Lu, R. Danzer, and F. D. Fischer, Phys. Rev. E 65, 067102 (2002).

[30] Y. S. Cheong, G. K. Reynolds, A. D. Salman, and M. J. Hounslow, Int. J. Miner. Process. 74, S227 (2004).

[31] E. W. Andrews and K. S. Kim, Mech. Mater. 31, 689 (1999).

[32] P. A. Cundall and O. D. L. Strack, Geotechnique 29, 47 (1979).

[33] B. K. Mishra and C. Thornton, Int. J. Miner. Process. 61, 225
(2001).

[34] C. Thornton and L. F. Liu, Powder Technol. 143-4, 110 (2004).

[35] C. Thornton, M. T. Ciomocos, and M. J. Adams, Powder Technol. 140, 258 (2004).

[36] D. O. Potyondy and P. A. Cundall, Int. J. Rock Mech. Min. Sci. 41, 1329 (2004).

[37] G. A. D'Addetta and E. Ramm, Granular Matter 8, 159 (2006).

[38] H. A. Carmona, F. Kun, J. S. Andrade, Jr., and H. J. Herrmann, Phys. Rev. E 75, 046115 (2007).

[39] L. D. Landau and E. M. Lifshitz, Theory of Elasticity, Course of Theoretical Physics Vol. 7, 3rd ed. (ButterworthHeinemann, London, 1986).

[40] H. J. Herrmann, A. Hansen, and S. Roux, Phys. Rev. B 39, 637 (1989).

[41] T. Pöschel and T. Schwager, Computational Granular Dynamics: Models and Algorithms (Springer-Verlag, Berlin, 2005).

[42] G. A. D'Addetta, F. Kun, E. Ramm, and H. J. Herrmann, in Continuous and Discontinuous Modelling of CohesiveFrictional Materials, Springer Lecture Notes in Physics Vol. 568, edited by P. Vermeer (Springer-Verlag, Berlin, 2001), pp. 231-258.

[43] G. Lilliu and J. G. M. Van Mier, Eng. Fract. Mech. 70, 927 (2003).

[44] D. C. Rapaport, The Art of Molecular Dynamics Simulation, 2nd ed. (Cambridge University Press, Cambridge, England, 2004).

[45] J. E. Bolander and N. Sukumar, Phys. Rev. B 71, 094106 (2005).

[46] M. Yip, Z. Li, B. S. Liao, and J. E. Bolander, Int. J. Fract. 140, 113 (2006)

[47] R. Mahmoodi Baram and H. J. Herrmann, Phys. Rev. Lett. 95, 224303 (2005).

[48] N. F. Mott, Proc. R. Soc. London, Ser. A 189, 300 (1946).

[49] F. K. Wittel, F. Kun, H. J. Herrmann, and B. H. Kroplin, Phys. Rev. E 71, 016108 (2005).

[50] D. L. Turcotte, J. Geophys. Res., [Solid Earth Planets] 91, 1921 (1986).

[51] R. P. Linna, J. A. Åström, and J. Timonen, Phys. Rev. E 72, 015601(R) (2005).

[52] J. A. Åström, R. P. Linna, J. Timonen, P. F. Moller, and L. Oddershede, Phys. Rev. E 70, 026104 (2004).

[53] H. Katsuragi, D. Sugino, and H. Honjo, Phys. Rev. E 70, 065103(R) (2004).

[54] F. Wittel, F. Kun, H. J. Herrmann, and B. H. Kröplin, Phys. Rev. Lett. 93, 035504 (2004). 